\title{
Atherosclerosis in cancer patients
}

\author{
Mladosievicova $\mathrm{B}^{1}$, Petrikova $\mathrm{L}^{1,2}$, Valaskova $\mathrm{Z}^{1}$, Bernadic $\mathrm{Jr} \mathrm{M}^{3}$, Chovanec $\mathrm{M}^{4}$, Mego $\mathbf{M}^{4,5}$, \\ Bernadic Sr M ${ }^{1}$
}

Institute of Pathological Physiology, Faculty of Medicine, Comenius University, Bratislava, Slovakia. bernadic.m@gmail.com

\begin{abstract}
Cancer-related mortality have been declining in the last decades. Approximately half of adults and more than two thirds of children oncological patients live longer than 5 years after diagnosis. However, this optimistic scenario has been counterbalanced by an increasing cardiovascular risk in cancer patients. Atherosclerotic damage has been underestimated in oncology practice for a long time, but recently a significant number of cancer patients with cardiovascular risk factors and serious artery disease during and after anticancer therapy has been reported. Complexity of atherosclerosis in cancer patients is challenging. Herein, we describe cardiovascular risk factors and pathophysiological mechanisms of atherosclerosis induced by selected classic chemotherapeutics, targeted cancer therapies, hormonal agents and radiotherapy and new clinical data regarding atherosclerosis, which received a particular attention in recent years (Tab. 1, Ref. 26). Text in PDF www.elis.sk.

KEY WORDS: cardiovascular disease, atherosclerosis, cardiotoxicity, risk factors, hypertension, hyperlipidemia.
\end{abstract}

\section{Introduction}

Advantages in early diagnosis and cancer therapy significantly contributed to cure or improved survival of oncological patients. Many of them die more frequently from cardiovascular diseases than from cancer relapse.

Multimodal anticancer therapy includes the use of several treatment options in order to be more effective. Beside surgery, radiotherapy, classic cytostatics and hormonal therapy, also targeted therapy and immunotherapy emerged in last decade.

Based on the results of the latest prospective clinical trials with long term (20-30 years) follow up of oncological patients, the attitude of medical community to cardiotoxicity has changed. Cardiovascular toxicity may cause transient functional disorders, but also a permanent irreversible damage of heart and vessels, including premature atherosclerosis (1-3).

${ }^{1}$ Institute of Pathological Physiology, Faculty of Medicine, Comenius University, Bratislava, Slovakia, ${ }^{2}$ Department of Oncohematology, Comenius University in Bratislava and National Cancer Institute, Bratislava, Slovakia, ${ }^{3}$ Department of Surgical Oncology, National Cancer Institute Bratislava, Bratislava, Slovakia, ${ }^{4} 2 n d$ Department of Oncology, Faculty of Medicine, Comenius University and National Cancer Institute, Bratislava, Slovakia, and ${ }^{5}$ Translational Research Unit at 2nd Department of Oncology, Faculty of Medicine, Comenius University and National Cancer Institute, Bratislava, Slovakia.

Address for correspondence: M. Bernadic, MD, PhD, Department of Surgical Oncology, National Cancer Institute, Klenova 1, SK-831 10 Bratislava, Slovakia.

Acknowledgements: This work was supported by the grant of Ministry of Education of Slovak Republic VEGA 1/0610/18.
Late effects of cancer therapy are those consequences, which are not present during the therapy, but occur years after completion of anticancer therapy. Late cardiovascular effects are:

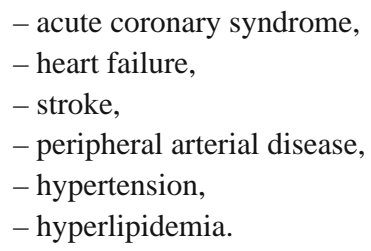

It is well established, that atherosclerosis is a lipid mediated inflammatory disease. However, the complex immune reactions driving inflammation in the vascular wall in response to an atherosclerotic microenvironment are still being unexplained (4). Not only anti-cancer therapy, but also malignant disease process itself can increase risk of atherosclerosis via several mechanisms with inflammation, prothrombotic state and oxidative stress playing the key roles. Cancer cells produce pro-inflammatory cytokines and chemokines, which can induce endothelial dysfunction, increased permeability in microvasculature and a leakage of pro-coagulation factors (such as tissue factor) and platelet-derived growth factor, what results in pro-atherosclerotic process. In oncological patients, additional stressors, e.g. surgical interventions and others contribute to the thrombosis. A more detailed knowledge of the common overlapping biological nature of cancer and cardiovascular diseases at the level of genes, signaling pathways and cells is a promise of new perspective therapeutic and preventive strategies for both disorders (5-7). 


\section{Cardiotoxic effects of classic anticancer agents with a focus on atherosclerosis}

The risk of stable angina pectoris, acute coronary syndrome and peripheral arterial disease was demonstrated to be associated with several classic chemotherapeutics, mainly cisplatin, paclitaxel, methotrexate, bleomycin, vinblastine and some other cytostatics (1). An impaired vasodilation, anti-thrombogenic and anti-inflammatory role of endothelium, arterial thrombosis, procoagulation status, hypotension and vasospasms belong to the pathomechanisms of acute coronary syndrom. Atherosclerotic damage in cancer patients can also be found in aorta, carotid, iliofemoral, mesenterial and limb arteries. Peripheral arterial disease can develop in the first months of therapy or as the late form delayed by several years after anticancer therapy.

Most data in relation to atherosclerotic damage is linked to cisplatin. Cisplatin is considered to be associated with acute and late cardiovascular complications. This substance induces vasospasm, increased aggregability of thrombocytes, increased levels of von Willebrand factor and hypomagnesemia. The cardiotoxicity of platinum derivatives can be also late $(1,8,9)$. Cisplatin used to be detectable in plasma 10-20 years after completion of therapy, while it could continually stimulate endothelium and increase the risk of atherosclerosis (10). It can also persist in multiple organs.

In relation to cisplatin, a special attention is given to cured oncological patients with a higher rate of cardiovascular risk factors and increased risk of premature atherosclerosis. The patients treated with the total dose of $850 \mathrm{mg}$ of cisplatin or higher had a 3 -fold higher rate of cardiovascular complications compared to the patients treated just surgically (10). The study of Meinardi et al observed in the group of patients aged 50 years of younger hypercholesterolemia in $79 \%$ of patients and hypertension in $39 \%$ of patients at the follow up 10 years after completion of anticancer therapy (11).

Cisplatin can cause a damage in several vascular territories. This drug may abolish capillary beds.

Increased risk of hypertension was found in testicular cancer survivors treated with cisplatin. In a Norway study of Sagstuen et al, with long-term follow up of 1289 testicular cancer survivors (median duration of 11.2 years) significant increase in age-, testosterone-, and body mass- adjusted systolic and diastolic blood pressure was observed. Compared with 2847 healthy males, the risk of hypertension was significantly higher in testicular cancer survivors (odds ratio 1.4, 95\% CI 1.2-1.7). The odds were highest for the cisplatin more than $850 \mathrm{mg}$ group (odds ratio $=2.4$; 95\% CI, $1.4-4.0$ ) (12).

The administration of bleomycin and vinblastine can contribute to endothelial damage.

\section{Cardiotoxic effects of targeted anticancer agents with a focus on atherosclerosis}

Targeted agents have more specific mode of action compared to classic chemotherapy. They aim at the targeted molecules found on the surface or inside cancer cells. There are 2 main groups of these agents - monoclonal antibodies and tyrosine kinase inhibitors (so called small molecules).

Although the concept of targeted therapy is based on more specific targeting of malignant cells, contrary to classic cytostatic therapy, it is important to state that the target structures play a physiologic role also in other structures of organism and their blockage outside tumor leads to a manifestation of several unexpected adverse effects. One of the most serious toxicity observed is cardiovascular toxicity (1).

Atherosclerosis induced by tyrosine-kinase inhibitors (TKIs) can be mediated by inhibited angiogenesis and mitochondrial dysfunction. Mitochondrial dysfunction leads to decreased levels of calcium and nitric oxide and increased production of reactive oxygen species. The risk of atherosclerosis can be accelerated also by TKI-induced hypertension, endothelial dysfunction, vascular smooth muscle cell dysfunction and activation/adhesion of inflammatory cells (13).

Hypertension is often associated with anti-angiogenic treatment - monoclonal antibody antiVEGF and TKI of VEGF receptor (VEGFR) (1). Bevacizumab, a monoclonal antibody blocking vascular endothelial growth factor (VEGF), can contribute to an acute coronary thrombosis by endothelial damage and inducing pro-coagulation status.

In the metaanalysis, which evaluated the rate of hypertension in prospective studies of nearly 5000 patients treated with TKI sunitinib due to renal carcinoma or other malignancy, the overall rate of hypertension was $22 \%$ and the rate of severe hypertension was $7 \%$ (2). However, the onset of hypertension during the therapy with TKI VEGFR can be also a predictor of better treatment response. A higher effectivity of sunitinib therapy was observed in patients who were treated due to hypertension. In patients treated with sorafenib, the rate of hypertension was mildly lower compared to sunitinib. The highest rate of hypertension was observed in association with axitinib. In the studies of more than 1900 patients, the incidence of hypertension was $40 \%$, while severe hypertension (grade 3 and 4) was observed in $13 \%$ of patients (2).TKI VEGFR therapy is associated with an increased risk of ischemia or myocardial infarction, cardiac dysfunction and heart failure. Although anti-angiogenic therapy with VEGF inhibitors are strongly associated with hypertension during therapy, the longterm effects of these drugs remain undefined. Also tyrosine kinase inhibitors targeting Abl and other proteins ponatinib and nilotinib can induce a progressive arterial damage, nilotinib especially on lower extremities and ponatinib in coronary and brain arteries (3, 7). Abl is protein necessary for the viability of endothelial cells, its inhibition by these agents can lead to vessel damage and progression of atherosclerotic process.

Cardiovascular risks associated with atherosclerosis following anticancer therapy are summarized in the Table 1 .

\section{Cardiotoxic effects of selected hormonal anticancer therapy with a focus on atherosclerosis}

In the context of androgen deprivation therapy (ADT), the question of an adverse effects of gonadotropin releasing hor- 
Tab. 1. Targeted anticancer agents associated with the risk for developing vascular toxicity (3).

\begin{tabular}{lccccc}
\hline & HTN & AP & MI & Stroke & PAD \\
\hline TKI & & & & & \\
Sorafenib & $\mathrm{X}$ & $\mathrm{X}$ & $\mathrm{X}$ & $\mathrm{X}$ & \\
Sunitinib & $\mathrm{X}$ & $\mathrm{X}$ & $\mathrm{X}$ & $\mathrm{X}$ & \\
Pazopanib & $\mathrm{X}$ & $\mathrm{X}$ & $\mathrm{X}$ & $\mathrm{X}$ & \\
Axitinib & $\mathrm{X}$ & $\mathrm{X}$ & $\mathrm{X}$ & $\mathrm{X}$ & \\
Regorafenib & $\mathrm{X}$ & $\mathrm{X}$ & $\mathrm{X}$ & & \\
Cabozantinib & $\mathrm{X}$ & & $\mathrm{X}$ & $\mathrm{X}$ & \\
Vandetanib & $\mathrm{X}$ & & & $\mathrm{X}$ & \\
Lenvatinib & $\mathrm{X}$ & & $\mathrm{X}$ & $\mathrm{X}$ & \\
Nilotinib & & $\mathrm{X}$ & $\mathrm{X}$ & $\mathrm{X}$ & $\mathrm{X}$ \\
Ponatinib & $\mathrm{X}$ & $\mathrm{X}$ & $\mathrm{X}$ & $\mathrm{X}$ & $\mathrm{X}$ \\
\hline
\end{tabular}

\begin{tabular}{|c|c|c|c|c|}
\hline \multicolumn{5}{|l|}{$\begin{array}{l}\text { Monoclonal } \\
\text { antibodies }\end{array}$} \\
\hline Bevacizumab & $\mathrm{X}$ & $\mathrm{X}$ & $\mathrm{X}$ & $\mathrm{X}$ \\
\hline Ramucirumab & $\mathrm{X}$ & $\mathrm{X}$ & $\mathrm{X}$ & $\mathrm{X}$ \\
\hline Rituximab & $\mathrm{X}$ & $\mathrm{X}$ & $\mathrm{X}$ & $\mathrm{X}$ \\
\hline
\end{tabular}

\begin{tabular}{|c|c|c|c|c|}
\hline $\begin{array}{l}\text { Fusion proteins } \\
\text { VEGF-receptor } \\
\text { ("VEGF traps") } \\
\text { Aflibercept }\end{array}$ & $\mathrm{X}$ & & $\mathrm{X}$ & $\mathrm{X}$ \\
\hline \multicolumn{5}{|l|}{ mTOR inhibitors } \\
\hline Everolimus & $\mathrm{X}$ & $\mathrm{X}$ & & \\
\hline Temsirolimus & $\mathrm{X}$ & $\mathrm{X}$ & & \\
\hline
\end{tabular}

Proteasome

inhibitors

\begin{tabular}{llll} 
Bortezomib & & $\mathrm{X}$ & $\mathrm{X}$ \\
Carfilzomib & $\mathrm{X}$ & $\mathrm{X}$ & \\
\hline
\end{tabular}

HTN - arterial hypertension, AP - angina pectoris, MI - myocardial infarction, PAD - peripheral arterial disease, VEGF - vascular endothelial growth factor, mTOR - mammalian target of rapamycin

mone (GnRH) agonists (e.g. leuprolide, goserelin), known also as luteinizing hormone releasing hormone (LHRH) agonists, on cardiovascular system become actual in the recent years. Cardiotoxicity is a special problem mainly in patients with pre-existing cardiovascular comorbidities and risk factors. One of the most important studies evaluating the cardiovascular risk associated with ADH was the observational study on 73196 men with prostate cancer (14). In patients treated with GnRH agonists, an increase of ischemic heart disease by $16 \%$, myocardial infarction by $11 \%$ and sudden cardiac death by $16 \%$ was observed compared to the patients without hormonal therapy. Also several newer studies in ADH patients confirmed an increased rate of myocardial infarction, stroke, thrombosis and other complications $(13,14)$. GnRH agonists` therapy has direct and indirect cardiovascular effects. The indirect mechanism of cardiovascular risk in ADH is related to increased weight, insulin resistance, dyslipidemia. The direct effect of ADT is manifested via cardiomyocytes, which express receptors for androgen, GnRH and FSH (15). ADT can cause an increased levels of LDL cholesterol, triglycerides and also induce inflammation and insulin resistance $(1,15)$. Until now, no trials have prospectively addressed the risks of coronary artery disease associated with ADT.
The metaanalysis by Carneiro et al in 2015, which included 13 studies ( $n=137$ 658), showed that ADT had significant adverse cardiovascular effects (16). ADT in prostate cancer patients (treated at least 6 months) increased the rate of acute myocardial infarction, stroke, thrombosis, heart failure and arrhythmias.

The hypothesis that GnRH antagonists act less harmfully on cardiovascular system compared to agonists is not yet confirmed (17). A comparison of GnRH agonists and GnRH antagonists` cardiotoxicity is the subject of intensive research and ongoing clinical studies.

Considering the significant use of ARTA (androgen-receptor targeted agents) enzalutamide and abiraterone acetate - in the therapy of castrate resistant prostate cancer, scientific literature started to pay attention also to their effects on cardiovascular system (18).

Aromatase inhibitors (anastrazole, letrozole, exemestan) used in therapy of breast cancer block the conversion of androgens to estrogen and have variable effects on lipids (19). An analysis of data from seven clinical trials with more than 30000 patients showed that longer duration of aromatase inhibitor use was associated with a statistically significant increase in the odds of hypercholesterolemia as compared with anti-estrogen drug tamoxifen (20). In addition, there may be mildly different effects on lipid profiles between aromatase inhibitors (19).

\section{Cardiotoxic effects of radiotherapy with a focus on athero- sclerosis}

It is well-known that radiotherapy (RT) is associated with an increased risk of carotid and coronary artery disease. Accelerated atherosclerosis and subsequent carotid stenosis are known vascular complications after radiation with doses > $50 \mathrm{~Gy}$.

Cases of atherosclerotic changes after RT with medium doses (30-50 Gy) are not fully understood. A radiation damage of carotid arteries can manifest with neurological symptoms (2).

RT may induce earlier atherosclerosis in any fields of radiation. Pathogenetic mechanisms include endothelial dysfunction, infiltration with monocytes, lymphocytes and macrophages and lysosome activation. The whole process can be accelerated by dyslipidemia, smoking, diabetes mellitus and hypertension (19). Late cardiovascular consequences of radiotherapy for Hodgkin lymphoma and breast cancer have been best studied $(19,21)$. Although a significant progress was made in the field of RT in recent years and modern special radiation techniques enable a minimization of normal tissue damage, results of many published studies are based on old outdated radiation treatment modalities.

Cardiologic complications induced by RT are extremely rare manifested during its application. More frequent and more serious are late consequence manifesting after a latent period $>1$ year after treatment completion. Cardiovascular damage caused by RT can progress over time after treatment completion.

The risk of damage is increasing with an increasing RT dose and a significant increase of cardiologic diseases was observed mainly after 35 Gy (1). At present it is obvious that also doses lower than 20 Gy can have a detrimental effect on heart. This is especially true in patients with risk factors. 
Patients with RT induced acute coronary syndrome (ACS) frequently have so called silent ischemia without typical manifestation of chest pain due to RT induced autonomous dysfunction or this syndrome is manifested atypically, as abdominal problems (nausea and vomiting) mainly in older patients and in childhood cancer survivors. The cumulative risk of myocardial infarction up to 30 years after RT is set at 10-13\% (2). Ischemic heart disease (IHD) can be manifested with a latent period 10-15 years after radiotherapy (2). Authors Girinsky et al in the prospective study evaluated 179 patients after Hodgkin lymphoma`s treatment, including RT of mediastinum, via a coronary angiography in the time interval 0.5-40 years after the treatment (median 9.5 years). Abnormalities in the coronary arteries were found in $15 \%$ of patients within first 5 years after RT and in $34 \%$ of patients with a delay of more than 10 years after RT. A severe stenosis required an intervention (stent or bypass) in $5.5 \%$ of patients. Cardiologic risk factors and overall radiation dose belong to independent prognostic factors (21).

The Oxford study of the authors Darby et al monitored the rate of serious coronary events in the cohort study of the participants treated due to breast cancer in the years 1958 - 2001. The incidence of serious ischemic complications increased by $7.6 \%$ linearly with each Gray of radiation delivered to the heart. Almost all patients in this study were treated with older radiation techniques. The increase started in the first 5 years after RT and continued also in the 3rd decade (22). Data published from the recent study of breast cancer patients treated from 2005 to 2008 with median follow-up of 7.6 years showed that each Gray of radiotherapy to the cardiac region was associated with a 16.5 percent increased risk of adverse events (23).

IHD belongs to serious outcomes also in patients with testicular cancer treated by RT of mediastinum. Authors van der BeltDuseboat et al demonstrated in testicular cancer survivors that mediastinal irradiation was associated with a 3.7-fold (95\% CI, 2.2- to 6.2-fold) increased myocardial infarction (MI) risk compared with surgery alone, whereas infradiaphragmatic irradiation was not associated with an increased MI risk (24). In this study the standardized incidence ratio (SIR) for MI was significantly increased in nonseminoma survivors with attained ages of less than $45(\mathrm{SIR}=2.06)$ and 45 to 54 years $(\mathrm{SIR}=1.86)$.

An increased incidence of hyperlipidemia was found among men treated for testicular cancer, which appears to be associated with RT and/or chemotherapy $(8,19)$.

\section{Cardiotoxic effects of allogeneic stem cell transplantation}

Dyslipidemia, impaired glucose tolerance and arterial hypertension can be induced also by allogeneic stem cell transplantation $(1,25)$. The whole body irradiation and intensive immunosuppressive therapy contribute to this as well. Authors Blaser et al performed a retrospective study of 761 patients who underwent allogeneic stem cell transplantation from 1998 to 2008 and survived at least 100 days. Seventy three percent of them developed hypercholesterolemia and 73 percent developed hypertriglyceridemia in the first two years (26).

\section{Conclusion}

Vascular effects of anticancer therapy is an area of intense ongoing investigation. Recent data provide the potential for a better understanding of molecular pathomechanisms of increased cardiovascular risk in cancer patients.

There are not enough clinical trials aimed at the benefit of screening of vascular complications associated with atherosclerosis in asymptomatic oncological patients.

In many cases, the possibility to positively influence these complications is undisputable. Although some individuals with no risk factors will experience premature atherosclerosis, other patients develop cardiovascular toxicity in the setting of underlying risk factors. Therefore, it is necessary to early identify high risk patients. Selected oncological patients are not rarely also candidates for revascularization. Development of new generally accepted guidelines for the screening and management of known risk factors and treatment with statin-based lipid lowering therapy, anti-platelet therapy, angiotensin-converting enzyme inhibitors, angiotensin receptor blockers, beta blockers and other agents could contribute to a better quality of life of these patients.

\section{References}

1. Mladosievičová B et al. Kardioonkologie. Praha: Grada Publishing a.s., 2014, 208 pp.

2. Zamorano JL, Lancellotti P, Rodriguez et al. ESC Committee for Practice Guidelines (CPG) 2016 ESC Position Paper on cancer treatments and cardiovascular toxicity developed under the auspices of the ESC Committee for Practice Guidelines: The Task Force for cancer treatments and cardiovascular toxicity of the European Society of Cardiology (ESC). Eur Heart J 2016; 37: 2768-2801.

3. Herrmann J1, Yang EH2, Iliescu CA et al. Vascular Toxicities of Cancer Therapies: The Old and the New-An Evolving Avenue. Circulation 2016; 133 (13): 1272-1289.

4. Ketelhuth DFJ, Lutgens E, Bäck M et al. Immunometabolism and atherosclerosis: perspectives and clinical significance- A position paper from the working group on atherosclerosis and vascular biology of the European Society of Cardiology. Cardiovasc Res 2019. pii: cvz166.

5. Koene RJ, Prizment AF, Blaes A, Konety SH. Shared risk factors in cardiovascular disease and cancer. Circulation 2016; 133 (11): 1104-1114.

6. Masoudkabir F, Sarrafzadegan N, Gotay $\mathbf{C}$ et al. Cardiovascular disease and cancer: Evidence for shared disease pathways and pharmacologic prevention. Atherosclerosis 2017; 263: 343-351.

7. Giza DE, Iliescu G, Hassan S et al. Cancer as a risk factor for cardiovascular disease. Curr Oncol Rep 2017; 19 (6): 39.

8. Chovanec M, Abu Zaid M, Hanna N et al. Long-term toxicity of cisplatin in germ-cell tumor survivors. Ann Oncol 2017; 28 (11): 2670-2679.

9. Valentová M, Mladosievicová B. Coronary heart disease and hypertension as late effects of testicular cancer treatment - a minireview. Klin Onkol 2011; 24 (1): 18-22.

10. Gietema JA, Meinardi MT, Messerschmidt J et al. Circulating plasma platinum more than 10 years after cisplatin treatment for testicular cancer. Lancet 2000; 355 (9209): 1075-1076. 


\section{$636-640$}

11. Meinardi MT, Gietema JA, van der Graaf WT et al. Cardiovascular morbidity in long-term survivors of metastatic testicular cancer. J Clin Oncol 2000; 18 (8): 1725-1732.

12. Sagstuen H, Aass N, Fosså SD et al. Blood pressure and body mass index in long-term survivors of testicular cancer. J Clin Oncol 2005; 23: 4980.

13. Brown SA, Nhola L, Herrmann J. Cardiovascular Toxicities of Small Molecule Tyrosine Kinase Inhibitors: An Opportunity for Systems-based Approaches. Clin Pharmacol Ther 2017; 101 (1): 65-80.

14. Keating NL, O'Malley AJ, Smith MR. Diabetes and cardiovascular disease during androgen deprivation therapy for prostate cancer. J Clin Oncol 2006; 24: 4448-4456.

15. Poljak Z, Hulin I, Maruscakova L, Mladosievicova B. Are GnRH and FSH potentially damaging factors in the cardiovascular system? Pharmazie. 2018; 73 (4): 187-190. doi: 10.1691/ph.2018.7992.

16. Carneiro A, Sasse AD, Wagner AA et al. Cardiovascular events associated with androgen deprivation therapy in patients with prostate cancer: a systematic review and meta-analysis. World J Urol 2015; 33: 1281-1289.

17. Albertsen PC. Androgen deprivation therapy and cardiovascular risk: No meaningful difference between GnRH antagonist and agonists. Eur J Cancer 2017; 87: 203.

18. Sternberg CN, Castellano D, Daugaard G et al. Abiraterone acetate for patients with metastatic castration-resistant prostate cancer progressing after chemotherapy: final analysis of a multicentre, open-label, early-access protocol trial. Lancet Oncol 2014; 15 (11): 1263-1268.
19. Ky B, Kondapalli L, Lenihan D. Cancer survivorship: Cardiovascular and respiratory issues - UpToDate 2019.

20. Amir E, Seruga B, Niraula $\mathbf{S}$ et al. Toxicity of adjuvant endocrine therapy in postmenopausal breast cancer patients: a systematic review and meta-analysis. J Natl Cancer Inst 2011; 103: 1299.

21. Girinsky T, M'Kacher R, Lessard N et al. Prospective coronary heart disease screening in asymptomatic Hodgkin lymphoma patients using coronary computed tomography angiography: results and risk factor analysis. Int J Radiat Oncol Biol Phys 2014; 89: 59-66.

22. Darby SC, Ewertz M, Hall P. Ischemic heart disease after breast cancer radiotherapy. N Engl J Med 2013; 368: 2527.2

23. van den Bogaard VA, Ta BD, van der Schaaf A et al. Validation and modification of a prediction model for acute cardiac events in patients with breast cancer treated with radiotherapy based on three-dimensional dose dcistributions to cardiac substructures. J Clin Oncol 2017; 35: 1171.

24. van den Belt-Dusebout AW, Nuver J, de Wit R et al. Long-term risk of cardiovascular disease in 5-year survivors of testicular cancer. J Clin Oncol 2006; 24 (3): 467-475.

25. Roziakova L, Setteyova L, Mistrik M, Mladosievicova B. Metabolic syndrome in patients after allogeneic hematopoietic stem cell transplantation. Lek Obzor 2015; 64 (5): 176-180.

26. Blaser BW, Kim HT, Alyea EP et al. Hyperlipidemia and statin use after allogeneic hematopoietic stem cell transplantation. Biol Blood Marrow Transplant 2012; 18: 575. 